CERN-PPE/96-36

7th March 1996

\title{
STUDY OF $\mathbf{K}_{s}^{0}, \Lambda$ AND $\bar{\Lambda}$ PRODUCTION IN S-W COLLISIONS AT $200 \mathrm{GeV} / c$ PER NUCLEON
}

\author{
The WA85 Collaboration
}

S. Abatzis ${ }^{1)}$, A. Andrighetto ${ }^{2, a)}$, F. Antinori ${ }^{2}$, R.P. Barnes ${ }^{3)}$, A.C. Bayes ${ }^{3}$, M. Benayoun ${ }^{4, b)}$, W. Beusch ${ }^{2}$, J.N. $\mathrm{Carney}^{3)}$, B. de la $\mathrm{Cruz}^{5)}$, D. Di Bari ${ }^{6}$, J.P. Davies ${ }^{3)}$, J.P. Dufey ${ }^{2}$, D. Elia ${ }^{6)}$, D. Evans ${ }^{3)}$, R. Fini ${ }^{6}$, B.R. French ${ }^{2)}$, B. Ghidini ${ }^{6}$, H. Helstrup ${ }^{7)}$, A.K. Holme ${ }^{7)}$, A. Jacholkowski ${ }^{2}$, J. Kahane ${ }^{4)}$, J.B. Kinson ${ }^{3}$, A. Kirk ${ }^{2}$,

K. Knudson ${ }^{2)}$, J.C. Lassalle ${ }^{2)}$, V. Lenti $\left.{ }^{6}\right)$, Ph. Leruste ${ }^{4, b)}$, J. Lien ${ }^{8)}$, R.A. Loconsole ${ }^{6)}$,

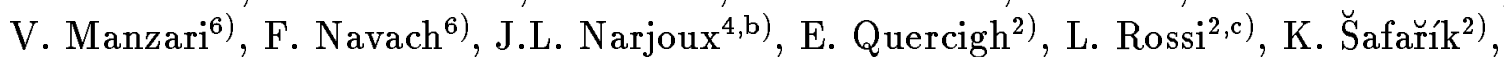
M. Sené ${ }^{4)}$, R. Sené ${ }^{4)}$, T. Storås $\left.{ }^{8}\right)$, G. Vassiliadis ${ }^{1)}$, M. Venables ${ }^{3)}$, O. Villalobos Baillie ${ }^{3)}$, A. Volte ${ }^{4)}$ and M.F. Votruba ${ }^{3)}$.

\begin{abstract}
Results on $\mathrm{K}_{s}^{0}$ production at central rapidity in sulphur-tungsten interactions are presented. The selection procedure used to identify $\mathrm{K}_{s}^{0}$ particles through their decay to two charged pions is described. The $m_{T}$ spectra for $\mathrm{K}_{s}^{0}, \Lambda$ and $\bar{\Lambda}$ and the relative production rates $\mathrm{K}_{s}^{0} / \Lambda$ and $\mathrm{K}_{s}^{0} / \bar{\Lambda}$, calculated in the kinematic region $p_{T}>1 \mathrm{GeV} / c$ and $2.5 \leq y_{L A B} \leq 3.0$, are discussed.
\end{abstract}

Submitted to Physics Letters B

\footnotetext{
1) Athens University, Nuclear Physics Department, GR-15771 Athens, Greece

2) CERN, CH-1211 Geneva 23, Switzerland

3) University of Birmingham B15 2TT, UK

4) Collège de France and IN2P3, F-75231 Paris, France

5) CIEMAT, E-28040 Madrid, Spain

6) Università di Bari and INFN, Sezione di Bari, I-70126 Bari, Italy

7) Universitetet i Bergen, N-5007 Bergen, Norway

8) Department of Physics, Universitetet i Oslo, N-0316 Oslo, Norway

a) Permanent address: Dipartimento di Fisica dell'Università, I-35131 Padua, Italy

b) Now at LPNHE, Univertité de Paris-6, France

c) Permanent address: Dipartimento di Fisica and Sezione INFN, I-16146 Genoa, Italy
} 
Heavy ion collisions provide, at present, the only way to study hadronic matter under extreme conditions of energy density in the laboratory. A new state of matter, the Quark Gluon Plasma (QGP), is expected to be created in central heavy ion collisions at relativistic energies. To investigate the possibility of QGP formation, many signals have been suggested as useful probes. In particular, the production of strangeness has been suggested to be of importance in understanding the dynamics of hadronic matter in relativistic heavy ion collisions $[1,2]$. This is due to the much shorter chemical equilibration time expected in a QGP compared to that in a Hadron Gas (HG) [1, 2]. Very little is known about the time constant of hadronization; in particular, mesons and baryons can be produced by different hadronization mechanisms, so that their relative abundances may not follow the laws of relative chemical equilibrium. Therefore it is also interesting to study strange meson production in order to compare with the production of strange baryons.

The CERN WA85 experiment [3] was designed to detect strange particle decays in relativistic heavy ion interactions in the central rapidity region with medium to high $p_{T}$ $\left(p_{T}>1 \mathrm{GeV} / c\right)$. The layout of the experiment has already been described in previous publications [4]. Here we just recall that its main component is a set of seven multiwire proportional chambers (MWPC) spanned over about $1.3 \mathrm{~m}$ length inside the OMEGA magnet, starting at $2.3 \mathrm{~m}$ from the target; the chamber cathodes have a modified butterfly shape [5] which allows them to detect only central rapidity, medium to high $p_{T}$ particles.

From this experiment we have already published results on hyperon $(\Lambda, \Xi, \Omega)$, antihyperon and charged kaon production in central sulphur-tungsten interactions at 200 $\mathrm{GeV} / c$ per nucleon $[6,7]$. In this letter we present results on $\mathrm{K}_{s}^{0}$ production and the relative yields $K_{s}^{0} / \Lambda$ and $K_{s}^{0} / \bar{\Lambda}^{1)}$ in the rapidity interval $2.5 \leq y_{L A B} \leq 3.0$ and $p_{T}>1 \mathrm{GeV} / c$. The results presented are based on 60 million sulphur-tungsten events.

Neutral strange particles have been identified by studying their decays into two charged particles, a topology called $\mathrm{V}^{0}$. The selections used to identify $\mathrm{V}^{0} \mathrm{~s}$ are described as follows. Any pair of oppositely charged particles gives a $\mathrm{V}^{0}$ candidate if:

(a) the distance of closest approach between the two tracks is less than $1 \mathrm{~cm}$,

(b) the angle between the vector momentum of the $\mathrm{V}^{0}$ and its apparent line of flight from the centre of the target is less than $0.75^{\circ}$,

(c) each track has at least four space points and traces through all seven MWPCs,

(d) the momentum of the $\mathrm{V}^{0}$ is greater than $6.5 \mathrm{GeV} / c$, and

(e) the reconstructed decay vertex and the other intersection of the two tracks in the bend plane projection are both in the fiducial interval of 125 to $230 \mathrm{~cm}$ from the target.

The meaning of the last condition is the following: the two tracks, traced in the magnetic field, normally cross twice in the bend plane (horizontal in the OMEGA magnet). Due to extrapolation errors, sometimes the vertical separation of the two tracks is not sufficient to recognize which of the two crossings is the true decay point of the $\mathrm{V}^{0}$ (see condition (a)). Selecting one of them as the actual $\mathrm{V}^{0}$ vertex could produce a bias in the

1) Since in our experiment it is not possible to recognize the electromagnetic decay $\Sigma^{0} \rightarrow \Lambda \gamma$, wherever in this letter we write $\Lambda(\bar{\Lambda})$, we mean the sum $\Lambda+\Sigma^{0}\left(\bar{\Lambda}+\overline{\Sigma^{0}}\right)$. 
particle yield because of the possible swapping of 'good' and 'wrong' crossings. This is particularly relevant for $\mathrm{K}_{s}^{0}$, for which the separation distance between the two crossings in the bend plane is larger than for $\Lambda \mathrm{s}$ (up to $80 \mathrm{~cm}$, in the $1.8 \mathrm{~T}$ OMEGA magnetic field, independently of the $\mathrm{V}^{0}$ momentum). Criterion (e) is robust against confusion between the two crossings, can be taken into account in the acceptance correction and does not affect the mass or momentum of the $\mathrm{V}^{0}$. In addition, it safely excludes the possibility that two oppositely charged tracks coming from the target cross again in the fiducial volume, simulating a $\mathrm{V}^{0}$. This criterion, important for good $\mathrm{K}_{s}^{0}$ selection, has also been applied to $\Lambda \mathrm{s}$ for purpose of comparison. More details about this procedure can be found in ref. 6 .

To identify $\mathrm{K}_{s}^{0}, \Lambda$ and $\bar{\Lambda}$, we require, in addition:

(f) $q_{T}>0.01 \mathrm{GeV} / c$, where $q_{T}$ is the transverse momentum of the decay tracks with respect to the $\mathrm{V}^{0}$ momentum. This selection removes the contamination coming from $\gamma \rightarrow e^{+} e^{-}$conversions,

(g) $|\alpha|<0.45$ for $\mathrm{K}_{s}^{0}$ candidates $^{2)}$, to eliminate $\Lambda$ and $\bar{\Lambda}$ contamination,

(h) $0.45<|\alpha|<0.60$ for $\Lambda$ and $\bar{\Lambda}$ candidates, in order to reduce the contamination from $\mathrm{K}_{s}^{0}$. An example of the Podolanski-Armenteros plot, where the meaning of the above selections is demonstrated, can be seen in fig. $2 \mathrm{~b}$ of ref. 9 .

The $\pi^{+} \pi^{-}, p \pi^{-}$and $\bar{p} \pi^{+}$invariant mass spectra obtained are shown in figs. $1 \mathrm{a}, \mathrm{b}, \mathrm{c}$, respectively. The shaded parts of fig. $1 \mathrm{~b}$ and fig. $1 \mathrm{c}$ show the $\mathrm{K}_{s}^{0}$ reflection in $p \pi^{-}$and $\bar{p} \pi^{+}$ mass spectra. Selecting $\Lambda$ and $\bar{\Lambda}$ candidates in a $50 \mathrm{MeV}$ mass interval centred on the $\Lambda$ mass [10] makes the $\mathrm{K}_{s}^{0}$ contamination negligible (about $1 \%$ in both cases). The $\mathrm{K}_{s}^{0}$ candidates have been taken in a $100 \mathrm{MeV}$ mass interval centred on the $\mathrm{K}_{s}^{0}$ mass $[10]$ and are free from $\Lambda$ and $\bar{\Lambda}$ contamination due to condition (g) above. The final sample consists of $10697 \mathrm{~K}_{s}^{0}, 22521 \Lambda$ and $5452 \bar{\Lambda}$ candidates.

The kinematic region of good acceptance for $\mathrm{K}_{s}^{0}$ is different from that for $\Lambda$ and $\bar{\Lambda}$ due to the different masses. In order to make a comparison, the yields and the transverse mass distributions have been computed in the overlapping region, namely $2.5 \leq y_{L A B} \leq 3.0$ and $p_{T}>1 \mathrm{GeV} / c$.

The transverse mass $\left(m_{T}=\sqrt{p_{T}^{2}+m^{2}}\right)$ distributions of $K_{s}^{0}, \Lambda$ and $\bar{\Lambda}$ are shown in fig.2. The distributions have been corrected for acceptance, unseen decay modes and reconstruction efficiency. The $\Lambda$ and $\bar{\Lambda}$ distributions have also been corrected for feeddown from $\Xi(\bar{\Xi})$ decays; the method has been described in a previous publication [11]. The spectra in fig. 2 have been fitted with the expression

$$
\frac{1}{m_{T}^{3 / 2}} \frac{\mathrm{d} N}{\mathrm{~d} m_{T}}=A e^{-\beta m_{T}}
$$

The inverse slopes $1 / \beta$ obtained are $219 \pm 3 \mathrm{MeV}$ for $\mathrm{K}_{s}^{0}, 239 \pm 3 \mathrm{MeV}$ for $\Lambda$ and $237 \pm 4 \mathrm{MeV}$ for $\bar{\Lambda}$, where the errors are statistical only. The inverse slope for $\mathrm{K}_{s}^{0}$ is compatible within one standard deviation with those for $\mathrm{K}^{+}$and $\mathrm{K}^{-}$, already published

${ }^{2)} \alpha$ is the Podolanski-Armenteros [8] variable $\alpha=\left(q_{L}^{+}-q_{L}^{-}\right) /\left(q_{L}^{+}+q_{L}^{-}\right) ; q_{L}^{+}$and $q_{L}^{-}$represent the positive and negative track momenta projected on the $V^{0}$ momentum. 
by our collaboration [7], and slightly smaller than for hyperons.

Table 1 shows the relative yields $K_{s}^{0} / \Lambda$ and $K_{s}^{0} / \bar{\Lambda}$ calculated in the full $y_{L A B}-p_{T}$ region used in this analysis and also for two different $m_{T}$ intervals: $m_{T}>1.5 \mathrm{GeV}$, which is the threshold value for $\Lambda(\bar{\Lambda})$, and $m_{T}>1.9 \mathrm{GeV}$ for comparison with $\Lambda(\bar{\Lambda})$ and $\Xi(\bar{\Xi})$ previously studied [4].

In ref. 12 a prediction is made for the production ratio $\mathrm{K}_{s}^{0} / \Lambda$ as a function of the ratio $\bar{\Lambda} / \Lambda$, in the framework of a thermal fireball model, assuming meson-baryon chemical equilibrium. Using the $\bar{\Lambda} / \Lambda$ value of $0.25 \pm 0.02$ obtained in the kinematic region used for the present analysis (with $m_{T}>1.9 \mathrm{GeV} / c$ and $2.5 \leq y_{L A B} \leq 3.0$ ), a $\mathrm{K}_{s}^{0} / \Lambda$ ratio between 0.12 and 0.14 is predicted, according to different assumptions about the central fireball (HG or QGP), its temperature and collective flow. Our results show a significantly higher ratio than that predicted. However, as pointed out in ref. 12 , due to the difference in the hadronization processes baryons and mesons do not need to be in chemical equilibrium relative to each other although chemical equilibrium may be established separately. Thus, the difference between the predicted $\mathrm{K}_{s}^{0} / \Lambda$ ratio and the one observed in our experiment can be interpreted as an indication for an off-equilibrium ratio of mesons to baryons in the strange sector.

The NA35 collaboration have published results concerning $K_{s}^{0}$ and $\Lambda(\bar{\Lambda})$ production in ultrarelativistic nucleus-nucleus collisions at $200 \mathrm{GeV} / c$ per nucleon [13]. Although their results are not directly comparable to ours because of different kinematic windows, a similar analysis [14] to that in ref. 12 has been carried out on S-S data from NA35. This analysis also indicates that strange mesons and baryons are not in full chemical equilibrium relative to each other.

In conclusion, we have reported results on the production of $\mathrm{K}_{s}^{0} \rightarrow \pi^{+} \pi^{-}$in central $\mathrm{SW}$ collisions at $200 \mathrm{GeV} / c$ per nucleon. The inverse slope of the $\mathrm{m}_{T}$ distribution is found to be $219 \pm 3 \mathrm{MeV}$, compatible with the values found for $\mathrm{K}^{+}$and $\mathrm{K}^{-}$[7]. The relative yields $\mathrm{K}_{s}^{0} / \Lambda$ and $\mathrm{K}_{s}^{0} / \bar{\Lambda}$ have been calculated in the three kinematic regions $2.5 \leq y_{L A B} \leq 3.0$ with $p_{T}>1 \mathrm{GeV} / c, m_{T}>1.5 \mathrm{GeV}$ and $m_{T}>1.9 \mathrm{GeV}$, respectively. 
[1] J. Rafelski and B. Müller, Phys. Rev. Lett. 48 (1982) 1066.

[2] P. Koch, B. Müller and J. Rafelski, Phys. Rep. 142 (1986) 167.

[3] WA85 Proposal, CERN/SPSC/84-76 P206 (1984), CERN/SPSC/87-18 P206 Add. (1987), CERN/SPSC/88-20 P206 (1988).

[4] WA85 Collaboration, D. Evans et al., Nucl. Phys. A566 (1994) 225c;

WA85 Collaboration, F. Antinori et al., Nucl. Phys. A566 (1994) 491c.

WA85 Collaboration, D. Di Bari et al., Nucl. Phys. A590 (1995) 307c.

[5] W. Beusch et al., Nucl. Inst. Meth. A249 (1986) 391.

F. Navach et al., Proceeding of the IX Autumn School The Physics of The QuarkGluon Plasma, Lisbon, 9-12 December 1987, Ed. J.Dias De Meus, S. Costa Ramos, World Scientific (1988), pp.103-105;

[6] WA85 Collaboration, S. Abatzis et al., Phys. Lett. B316 (1993) 615;

WA85 Collaboration, S. Abatzis et al., Phys. Lett. B347 (1995) 158;

WA85 Collaboration, S. Abatzis et al., Phys. Lett. B359 (1995) 382;

[7] WA85 Collaboration, S. Abatzis et al., Phys. Lett. B355 (1995) 401;

[8] J. Podolanski and R. Armenteros, Phil. Mag. 45 (1954) 13.

[9] WA85 Collaboration, S. Abatzis et al., Phys. Lett. B244 (1990) 130;

[10] Review of Particle Properties, Phys. Rev. D50, (1994) 1173.

[11] WA85 Collaboration, S. Abatzis et al., Phys. Lett. B270 (1991) 123;

[12] J. Letessier et al., Phys. Rev. D51 (1995) 3408;

[13] NA35 Collaboration, T. Aber et al., Z. Phys., C64 (1994) 195;

[14] J. Sollfrank et al., Z. Phys., C61 (1994) 659; 
Table 1. Meson to baryon production rates in $\mathrm{SW}$ interactions at $200 \mathrm{GeV} / c$ per nucleon.

\begin{tabular}{|c|c|c|c|}
\hline Ratio & $p_{T}>1 \mathrm{GeV} / \mathrm{c}$ & $m_{T}>1.5 \mathrm{GeV}$ & $m_{T}>1.9 \mathrm{GeV}$ \\
& $2.5<y_{L A B}<3.0$ & $2.5<y_{L A B}<3.0$ & $2.5<y_{L A B}<3.0$ \\
\hline & & & \\
$\mathrm{K}_{s}^{0} / \Lambda$ & $1.43 \pm 0.10$ & $0.25 \pm 0.02$ & $0.22 \pm 0.02$ \\
$\mathrm{~K}_{s}^{0} / \bar{\Lambda}$ & $6.45 \pm 0.61$ & $1.16 \pm 0.08$ & $0.87 \pm 0.09$ \\
& & & \\
\hline
\end{tabular}


Figure 1. The invariant mass spectra of a) $\left.\left(\pi^{+} \pi^{-}\right), b\right)\left(p \pi^{-}\right)$and c) $\left(\bar{p} \pi^{+}\right)$after the selections described in the text.

Figure 2. Transverse mass distributions for $\mathrm{K}_{s}^{0}, \Lambda$ and $\bar{\Lambda}$ candidates. The $\mathrm{K}_{s}^{0}$ data have been divided by 2 in order to avoid graphical overlap with the others. 

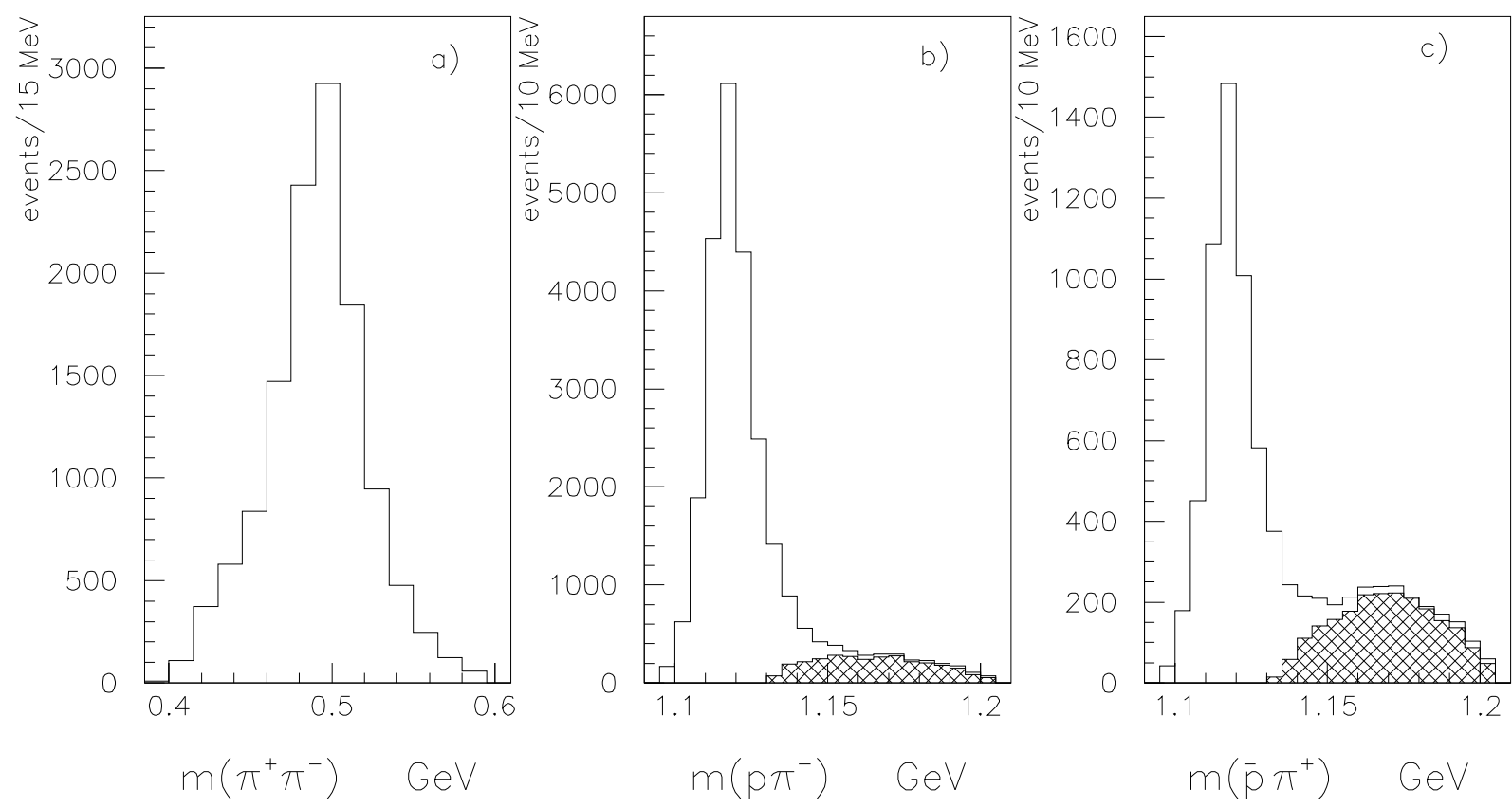

Figure 1. 


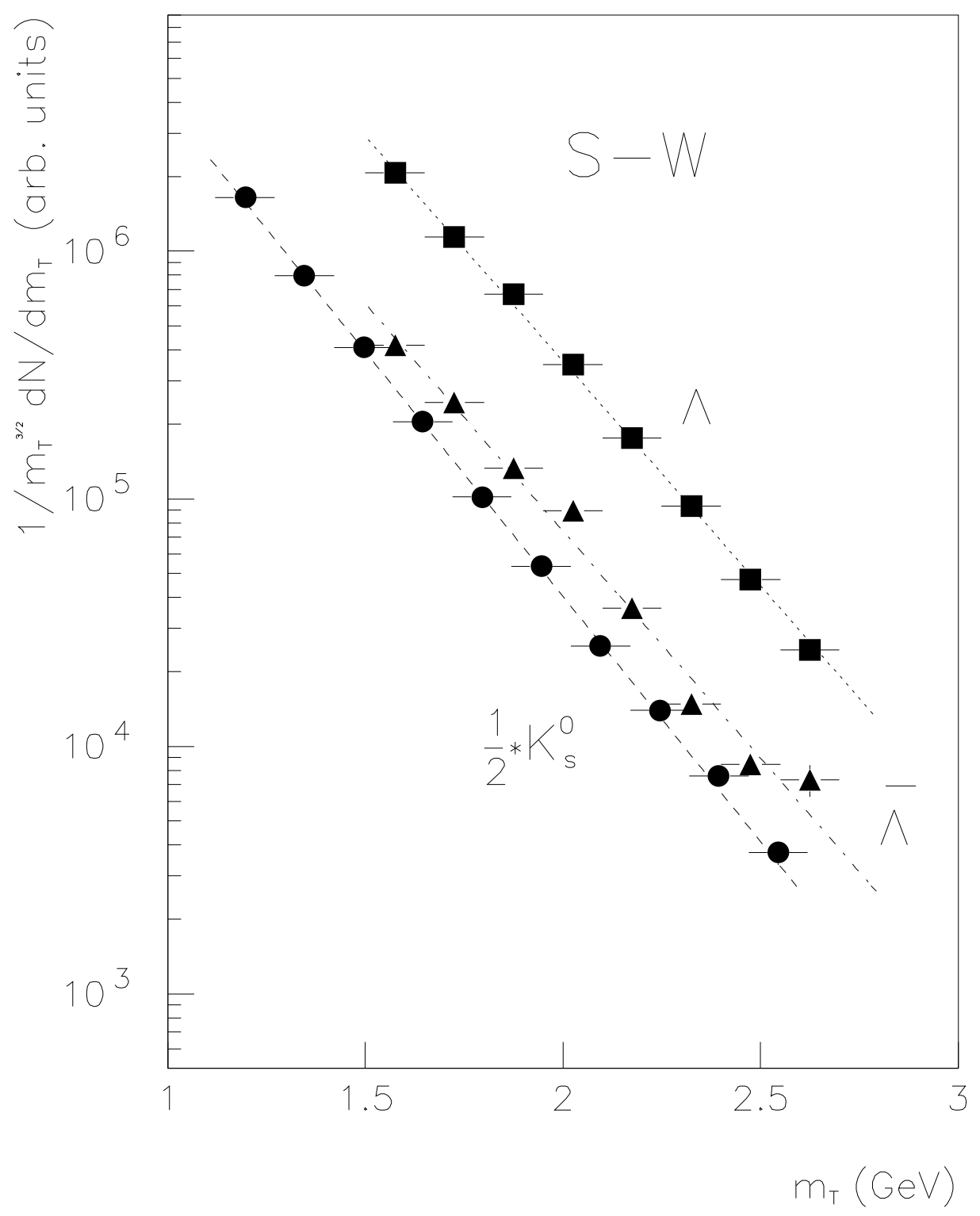

Figure 2. 